\title{
Can a Table Regulate Participation in Top Level Managers' Meetings?
}

\author{
Dyna Mlotkowski \\ Banque Priveé Edmond de \\ Rothschild \\ Geneva, Switzerland \\ dmlotkowski@bper.ch
}

Flaviu Roman

Ecole Polytechnique Fédérale

de Lausanne (EPFL)

Lausanne, Switzerland

flaviu.roman@epfl.ch

\author{
Stefano Mastrogiacomo \\ Université de Lausanne \\ (UNIL), \\ Lausanne, Switzerland \\ smastrog@unil.ch
}

\author{
Frédéric Kaplan \\ Ecole Polytechnique Fédérale \\ de Lausanne (EPFL) \\ Lausanne, Switzerland \\ frederic.kaplan@epfl.ch
}

\author{
Pierre Dillenbourg \\ Ecole Polytechnique Fédérale \\ de Lausanne (EPFL) \\ Lausanne, Switzerland \\ pierre.dillenbourg@epfl.ch
}

\begin{abstract}
We present a longitudinal study on the participation regulation effects in the presence of a speech aware interactive table. This study focuses on training meetings of groups of top level managers, whose compositions do not change, in a corporate organization. We show that an effect of balancing participation develops over time. We also report other emerging group-specific features such as interaction patterns and signatures, leadership effects, and behavioral changes between meetings. Finally we collect feedback from the participants and analyze qualitatively the human and social aspects of the participants interaction mediated by the technology.
\end{abstract}

\section{Categories and Subject Descriptors}

D.5.3 [Group and Organization Interfaces]: Collaborative computing, Computer supported cooperative work, Synchronous interaction; H.5.2 [User Interfaces]: Graphical User Interfaces

\section{General Terms}

Experimentation, Human Factors

\section{Keywords}

Meetings, Visualization, Group Evolution, Human Computer Interaction

\section{INTRODUCTION}

A large amount of effort has been devoted lately to meetings techniques and tools, to improve their effectiveness in

Permission to make digital or hard copies of all or part of this work for personal or classroom use is granted without fee provided that copies are not made or distributed for profit or commercial advantage and that copies bear this notice and the full citation on the first page. To copy otherwise, to republish, to post on servers or to redistribute to lists, requires prior specific permission and/or a fee.

GROUP'12, October 27-31, 2012, Sanibel Island, Florida, USA.

Copyright 2012 ACM 978-1-4503-1486-2/12/10 ...\$15.00. light of the increase of costs and decrease of productivity visible in many meetings. In this study, we evaluate the use of a previously developed technology called the Reflect Table [1] bundled with a new meeting analysis software (ReflectVisualizations), which we use as awareness and support tools for groups of top level managers in training sessions. We present new results of the influence of this technology and some insights about group dynamics over time. The outcomes of this new "real world" study are also checked against the outcomes of previous studies of the Reflect Table in controlled environments of undergraduate students' face to face encounters. We measure speech participation awareness and analyze its effects on balancing, by making this information available to the participants during the meeting, in the form of ratios of speech times. This is done without suggesting any form of regulation. Rather, the group members, when presented with this type of evidence, are responsible for applying self-regulation if and how they see fit, while meeting researchers can observe and analyze if the presence of this extended knowledge about their habits did trigger behavioral changes or not. Therefore, the group is responsible for its own regulation criteria.

\subsection{Motivation. Balanced Participation.}

In all organizations, face to face meetings are ubiquitous events. However, many of them fall short of their intended goals, and when it comes to collaborative learning, unbalanced participation leads to poor outcome for some attendants. A comprehensive analysis by Romano and Nunamaker [18] quantitatively breaks down the meetings problems and outcomes, with the goal of identifying and classifying practices and causes that lead to the sub-optimality of the meetings outcomes with respect to their goals. Among these are participation imbalance, overdue lengths due to poor time management, impaired agenda management, suboptimal preparation or lack of appropriate participants skills in conducting efficient meetings.

Balanced participation is thus one of the factors that lead to poor performance of meetings, and it is the chosen topic of interest for this study. Papers by Salomon [19], and Webster [22] discuss about socio-psychological causes that risk prevent team members from engaging equally into collaborative activities. In terms of learning, Cohen [9] investigates 
productivity and outcomes, concluding that collaborative exchanges are necessary for conceptual learning effectiveness, and that the more individuals participate, the more they learn. Hoyles [13] shows that verbalization is important in formation and fixation of concepts in the context of learning, therefore reduced participation can negatively impact the learning gain. Also, Huber [14] claims that both in the context of trainings and of problem solving, adequate information sharing plays an essential role because existing participants holding critical information or expertise but not sharing it will yield substandard aftereffects of those meetings.

Bettenhausen [7] studied the development of norms in newly formed groups, and found that members use their past experiences in similar social settings as scripts for choosing behaviors in their current situation. Another important contribution in what regards the way individuals in groups influence each other is done by Hackman [12] who concludes that ambient stimuli, discretionary stimuli and structure of group norms represent types of influences on individuals.

\subsection{Related Work}

Various academic researchers have devoted themselves to creating technological tools to assist the meeting process, both online (during the meeting) and offline (as post-meeting visualization of meeting data). The Meeting Mediator [16], which requires users to wear a sociometric badge, and the Agent Augmented Meeting [11], which creates virtual participants are such tools, and while researchers have proved their utility, we consider these methods to be too intrusive and interrupt the natural flow of the interaction in a meeting. We intend to follow a less-intrusive direction, of soft computing, latent technology and background or peripheral vision displays. In these lines, a metaphoric group mirror system was developed by Streng [21] that uses image metaphores to present insights from discussions. Similar work has been done by Bergstrom and Karahalios [5], whose time visualization patterns have the advantage of showing time evolution, but whose sizes are difficult to assess and matching a color with its corresponding participants is a challenge. In a similar manner, they also designed a contribution value voting system [6]. Skog, Ljungblad and Holmquist [20] produced a visualization system, where the data visualized contains, among other information, reports about speech time in meetings; they project these images on on a vertical space or wall. DiMicco [10] created various types of information displays and evaluated their impact on the participants, finding that introduction to the display and no feedback redundancies were the most valued properties.

Among offline meetings support and analysis or data retrieval tools we can mention Nijholt's Meeting Information Visualization [17], and Hunter's MemTable [15]. Some of these solutions are still constrained by the portability of the technology, i.e. data can only be obtained from meetings held in a special room.

Most of these works suffer from one shortcoming present in our previous works as well: the lack of a real world study. This paper capitalizes on this by studying and evaluating our technology for the first time in the wild.

\subsection{Paper Structure}

The remainder of the paper is structured as follows: Section 2 describes the technology we used to support this user study of meetings, including the new modules and increments from previous versions (a new graphical visualization tool for post-meeting support and analysis), previous results in controlled environments of graduate students, and the methodology used in this study. Section 3 dissects the results and goes in-depth into analyzing the observations, while sections 4 and 5 discuss future development directions and conclusions of this research.

\section{TECHNOLOGY AND METHODOLOGY}

The Reflect Table [1] (Fig. 1) was created to be a background, unobtrusive and normative-neutral tool for in meetings participation awareness. The aim is to only be a mirror of the group interaction, presenting objective data, and remaining completely neutral in terms of judging the quality of discussions or group cooperation.

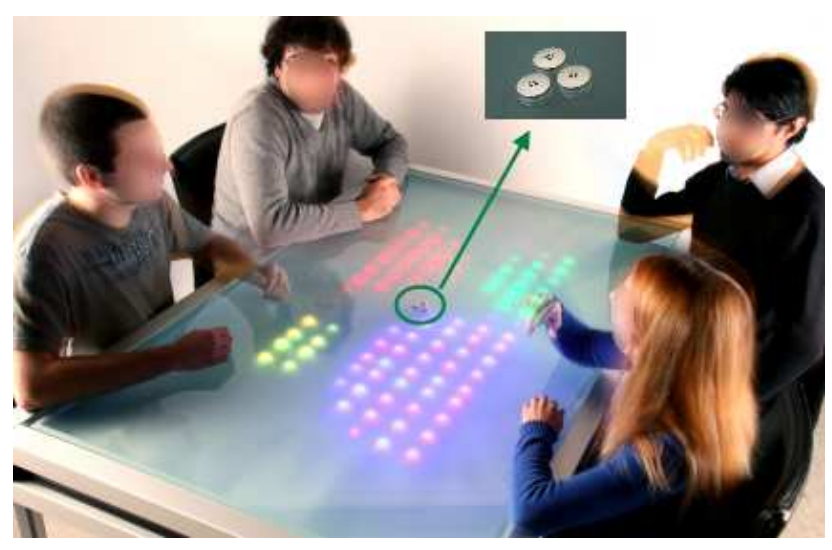

Figure 1: Reflect Table (from [1])

Unobtrusiveness is a key factor of the design aimed at keeping the communication flow as natural as possible. As opposed to private displays, the shared semi-ambient display was preferred for its capacity of integration into the table surface, and to relieve the participants from actively seeking information. This way, a breakdown of the speech times is presented in background of the active view area. The shared display reinforces the group mirror characteristic of the table. Participants have access to the values of speech time relative to each other, as well as to the link between the identity of each speaker and its relative speech time. The identity recall proves to be an important element in the qualitative assessment of the study.

\subsection{Reflect Table: Technical Description}

The technology relies on a physical 6-persons shaped table. Inside it contains a computer with input for speech capturing by means of a triangle array of microphones, and two special led panels with a total of 16x8 LEDs for output display (Fig. 1).

The LEDs use a technology that allows them to light up in different colors, based on a command sent through a device driver. The LED panels are used for dis-playing real-time meeting information. Each user has his/her corresponding color based in front of his position. The panels are smaller than the area of the table, so participants can still make use of paper documents or physical objects without disrupting the visibility of the LEDs. This display has several selectable 
modes, and in our current study we are using two of them: a hidden mode (nothing displayed while engine still running), and a territorial mode (Fig. 1). In this mode the LEDs display a territory in front of each participant, which accounts for the total speech ratio of his/her speech time with respect to the total detected speech. The participants can switch modes by gently touching the microphones on the top, which generates a recognizable waveform interpreted as a command.

The microphone array uses a triangulation algorithm that detects the speaker's direction based on comparing the time shifts of speech detected by the three microphones, and using this information to reconstruct the direction. The central placement of the microphones ensures that the space in front of the participants is freed from technical elements, thus they can use it for their natural interaction [4]. Another advantage of this design is that the time consistency of detection is guaranteed by the detection of a unique speaker from multiple sources.

The microphones acquire the signal, retain the speech duration from each direction (identifying each participant), and pass the waveforms to a speech features detection module [8] which computes some voice features (like pitch and its variance, intensity, speech rate, etc.). An average of the normalization of these 4 features is then used to compute the instant voice arousal or engagement. Apart from displaying a synthesis using the LEDs, the table is also a logger, by saving information to data files which are only kept internally, therefore offering no online connectivity.

\subsection{Reflect Visualizations: Meetings Analysis Software}

There are some inherent limitations that arise from the Reflect Table's creation as intelligent furniture, and from its design which targets a very specific setting. Also the previous studies did not benefit from support for complex offline analysis of data (which was done manually). The table itself cannot act as a comprehensive post-meeting display surface because it only has a few output LEDs, which do not offer the appropriate resolution required by such displays. Also, currently there is no study in an uncontrolled environment use of the Reflect Table, which is covered by this paper for the first time.

We came to the creation of a software that we called ReflectVisualizations to address the offline visualization limitations of the Reflect Table. We acknowledged the need for a more automatized analysis tool (as opposed to manually using Excel or other software able to produce visualizations), as a module that can automatically process the table's specific logs to help distinguish characteristics that are easier to observe from visualizing data in a graphical form. The purpose of this tool is to be bundled with the Reflect Table to enable more in-depth analysis of meetings, and capacitate group evolution studies, by integrating data from several meetings with the same attendance composition.

\subsubsection{Concept}

We designed the software as a leverage for the meetings analysis we perform, but also to be distributed to different categories of people involved in the process, namely the facilitators (if any) and the participants themselves, taking into account the need for interoperability, scalability, and dissemination requirements. The tool was created using $\mathrm{C \#}$ on .net platform, using several custom made controls along with other open source libraries, including the complex ZedGraph library for evolution plots. Other plots are made by an engine drawing directly on the canvas.

We chose to split the available frame into three sectors, according to the type of visualization we wanted to display. The following types were created: table replay, which aims to reproduce the display on the table, table syntheses, which shows in a graphical way some interaction features, and evolution graphs, which show the evolution of interaction on several scopes.

\subsubsection{Table Replay and Syntheses}

The upper left part of the ReflectVisualization screen shows a reconstruction of the table display in time. Here one can review the display of the table at any chosen moment, which we implemented as a video replay tool, as shown in Figure 2. This mode is typically useful for group support within debriefing sessions that may be organized to discuss their meeting practices.
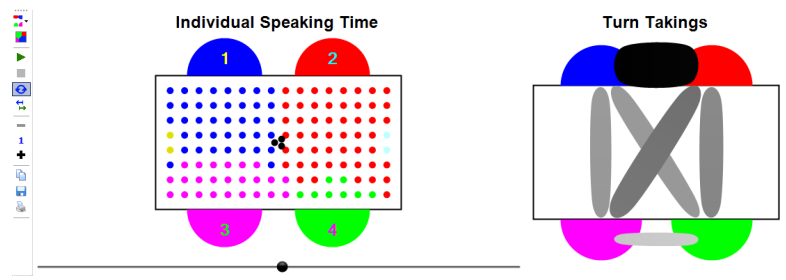

Figure 2: Table Replay and Synthesis Display

The upper right part is the summary display which computes other synthetic meeting features. They are created to overcome the limitation of the table display restricted to LEDs, and can show new syntheses that are available from the recorded data. Here we display the Turn Takings (Fig. 2) to show who spoke more after who (which we view as an indication of pair speech and dual interactivity). The drawing consists of pairwise lines whose gradient point out the number of speech acts transfers from one person to another, and where the darker color suggests a higher number of transfers.

\subsubsection{Evolution Graphic and Report}

The most important part of the visualization tool is the graphical evolutionary form of presenting the data (Fig. 3). This is the main added value of the tool, to make available information in a graphical, evolutionary representation. The participants colors are the same as the ones used on the Reflect Table display. The X coordinate represents each minute of the meeting (either relative to the beginning or absolute time of day), while the Y coordinate represents the fraction of a minute that the participant was recorded to produce speech chunks.

The plots use evolution curves for each participant, and they can be smoothened by moving-averaging values over more minutes. A scattered plot is also available, a total speech histogram can be created, and a dashed line can be displayed to correlate the moment chosen on the Replay sector with the time in this graphic.

We acknowledge that often managers do not have the time to look deeper into the analysis provided by the tool, and debriefing sessions may not be organized after each session. 


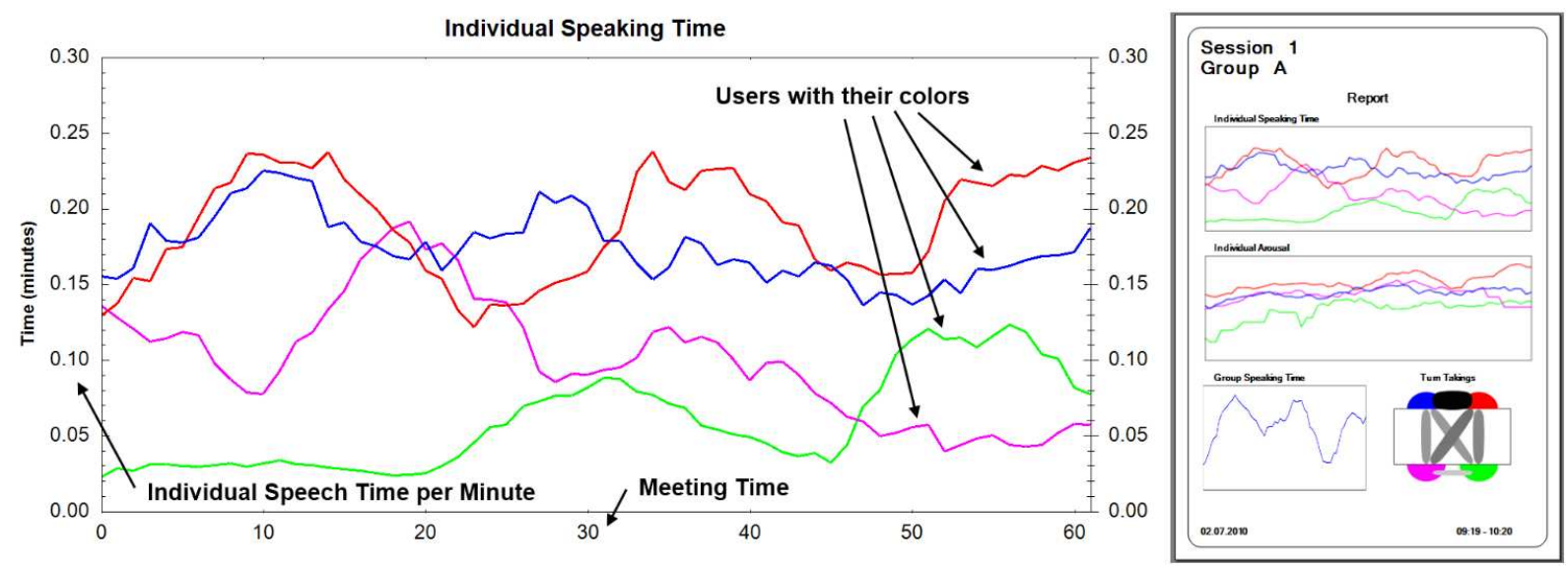

Figure 3: Reflect Visualizations: Evolution Graphic and Report

Therefore we conceived a one-snapshot summary in the form of a group PDF Report (Fig. 3) which shows the evolution of individual and group measures, as well as turn takings, and represents, for the participant, a first glimpse at their meeting dynamics. The report is aimed to be distributed to each group after each session. We maintain the principle of objectively presenting data only, and do not include any comments about the developments in the meeting.

\subsection{Previous Experiments: Results and Limi- tations of the studies}

The Reflect Table has been previously used only in controlled environments [2, 3], producing encouraging results. Scenarios for studies included analyzing groups with the Reflect Table versus control groups without a Reflect Table, comparisons of displays such as the type of display used (hidden or territories), or displays of speech times of individuals versus speech times per topics (a Wizard-of-Oz technique was employed where a scientist would silently and inadvertently attend the meeting and mark the changes in the topics).

The participants were undergraduate IT students that were paid for the experiment, and asked to solve a murder mystery given as a hidden profile task, where pieces of information were either shared among booklets or available only in one of the booklets. The students were constrained to read only from their copy and not allowed to physically exchange the booklets. This type of task inherently requires the participants to collaborate to share the information, for the correct identification of a perpetrator. The students were not informed of and there was no mentioning of any theoretical benefit from balanced participation. The measurements included participation time and discussion time per topics, and a questionnaire at the end of the meeting was aimed at collecting qualitative data about the participants' perspectives.

The results showed that in the absence of participation information, the participants were not able to correctly identify ratios of participation. More than $95 \%$ of the participants reported looking at the table for information, with only less than $25 \%$ claiming to be distracted by it, which ensures the design is appropriate. Further findings show that for extreme over participators (dominants or floor-monopolizers) the availability of the speech time information had an effect of determining them to reduce their speaking time and free the floor. However, no significant results were obtained for under-participators, except that all extreme under participators reported in the questionnaire that they did not consider equal or balanced participation to be important in any meeting.

A certain difference between previous studies and the current one rests in the fact that all previous studies were done on groups that were meeting a single time, whereas in the current study, we have the opportunity to observe teams meeting several times and evaluate the evolution of their behavior. Also, previous studies did not benefit from the availability of Reflect Visualizations or from debriefings.

\subsection{Methodology: A Real World approach}

The main research goal of this paper is to expand the study of this speech awareness tool in meetings to verify whether there is an added value and benefit in real world as well, after the incipient studies brought promising results. We want to see whether teams of top level managers undergoing training reunions, with the purpose of enlarging their expertise by sharing knowledge, experience the same behavioral changes (e.g. floor monopolizers release it and end their turn when becoming aware of over-participating) as students in a laboratory-organized experiment. The degree of success and the experience

This effort is the first assessment of a Reflect Table in an uncontrolled, real world situation. The setup is a training activity that involves 6 teams or groups, labeled from A to $\mathrm{F}$, each one composed of 5 or 6 people, undergoing multiple meetings with the Reflect Table, and having access to the ReflectVisualizations and to the report page after every meeting. There are a total of 10 meetings for each group (taking place roughly every month for a total period of one year), and the participants plan and schedule the sessions themselves at this frequency. The groups are composed of peer top level managers, but are heterogeneous in the sense that they manage distinct divisions in a large corporate organization. The teams were built in such a way that the attendants did not know each other before the start of the trainings. The members of the groups never change throughout the unfolding of the sessions. The collected speech time 
and features data is completely content-free and text confidential, the table can only log the speech time based on the direction of the sound. The overall content of their discussions is unknown, but the target of the training framework is to improve their mutual knowledge by exchanging individual work experiences about given topics (which are also unknown).

We only require each participant to use the same position at the table during all their planned group meetings, therefore ensuring consistency in the relationship between the color on the table and identity of each participant. We label the participants with numbers (1-6) and corresponding colors based on the table position (Blue, Red, Fuchsia, Green, Yellow, White). The groups have a coordinator who is managing the sessions, without participating herself in the meetings, and which ensures that the participants receive and read at least the reports of the sessions.

A short first encounter (Session 1) was organized without the Reflect Table with the goal to briefly introduce the participants to the purpose of the training and to each other. From the second session, the groups started using the Reflect Table in a specially designed room. Before the beginning of this session, they were given an introduction to the table, its features, and how to change the display mode. Besides touching the microphones to select this display mode, there is no other physical interaction of the participants with the table. From there, the teams are left alone and completely autonomous to devise their styles of interaction. Regarding the display mode, we advised them to use a hidden mode for the first two sessions, and a territory mode for the rest (the two values of display modes are one independent variable of the experiment). Post-meeting questionnaires were created for some meetings and distributed by the facilitator, but the response rate to any such questionnaires was out of our control. Overall we considered that this user study is done in an uncontrolled environment because we had no access to any other types of measurements, or control parameters.

\section{RESULTS}

To date, we have collected and analyzed data from meetings spanning over 6 sessions (sessions 2 to 7 ). We did not receive data from all the sessions, due to participants forgetting to turn the table on in some instances, schedule conflicts that forced some sessions to be held in a different room without the Reflect Table, and, in one case, a software problem that prevented meaningful data to be gathered during that session. In total, we procured data from 27 meetings, with a per-group collection of data from 4 to 6 sessions, depending on the group. We have confirmation that the first two meetings were held using the hidden mode, while the rest used the territories, as we recommended. At the end of each session held in hidden mode, they switched to the territories mode and discussed for about one minute about whether the result was expected or was a surprise.

\subsection{Quantitative Data Analysis}

We analyze the data gathered from the groups using the ReflectVisualization tool. We used a per-group data merging to put together all data from these sessions for each group.

\subsubsection{A metric for participation imbalance: When visual instant feedback is present, there is reg- ulation over time}

We defined and computed a measure of imbalance of the participation. In the process of defining this notion we considered a certain number of properties that should build up the definition, and also some issues required for keeping the measurement consistent throughout different sessions and different groups:

1. The measure is inversed-scale, meaning that the lower the value is (towards zero), the better. A value of zero indicates equal speech time among all participants

2. The sessions have a random duration, therefore the influence of number of minutes of each session should be avoided

3. The group (overall) speech time per minute varies among sessions, therefore it should not influence the result

Thus we defined the imbalance as the Session-averaged sum of absolute deviations of per-minute speech time of each participant, or more formally given $N$ as the participants number, $M$ as session time in minutes, and $X_{i j}$ as the speech time of participant $i$ in the minute $j$ of the session, the average session imbalance over all groups is defined as

$$
I=\frac{1}{M} \sum_{j=1}^{M} \sum_{i=1}^{N} A b s \operatorname{Dev}\left(X_{i j}\right)
$$

where

$$
\operatorname{Abs} \operatorname{Dev}\left(x_{i}\right)=\left|x_{i}-\frac{1}{N} \sum_{k=1}^{N} X_{k}\right|
$$

therefore giving the final formula of

$$
I=\frac{1}{M} \sum_{j=1}^{M} \sum_{i=1}^{N}\left|X_{i j}-\frac{1}{N} \sum_{k=1}^{N} X_{k j}\right|
$$

We used the averaging over the total duration of the meeting to overcome the random times of the sessions. We applied the formula to all meetings, and then averaged the results of each group on the same meeting number. When we had missing data (no sessions), the missing value was just discarded from the average.

By plotting the values obtained for each meeting (averaged over all groups), we notice the evolution of Imbalance, as shown in Fig. 4. The immediate visible result is that there is no effect in the hidden mode, however a balancing effect becomes visible in time with the use of the territories mode. It is difficult to decipher why the imbalance actually grew from the first hidden session to the second, but a tentative explanation could take into account the presence of the instructor to introduce the table at the beginning of the second session, revealing the ideas of participation equilibration, or the need to share more equally in the early context of getting to know each other better.

The effect of balancing of participation over time new and encouraging, and although the number of groups $(\mathrm{G}=6)$ and number of meetings $(\mathrm{M}=6)$ are insufficient for a sound statistical analysis of the phenomenon, we are optimistic to believe that possessing such a speech time awareness tool does help groups balance their participation over time. 


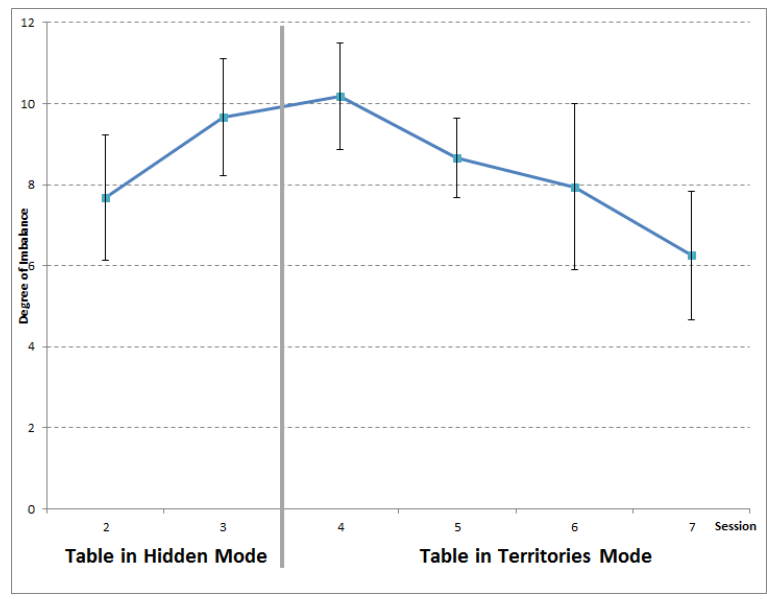

Figure 4: Evolution of Degree of Imbalance. A lower value means more balanced participation.

\subsubsection{Speech Time and Leadership: No leader vs. One leader}

Information on leadership is useful to shape the internals of the groups. We define and use the notion of leader based on a combination between their duration of speech and interactivity. We consider the assumption that those who want to share more could have more knowledge and hence become a higher authority for the group. What we observed by analyzing speech is that there are different types of leaderships. For example, in group D, there is no consistent leader throughout the sessions (Fig. 5).

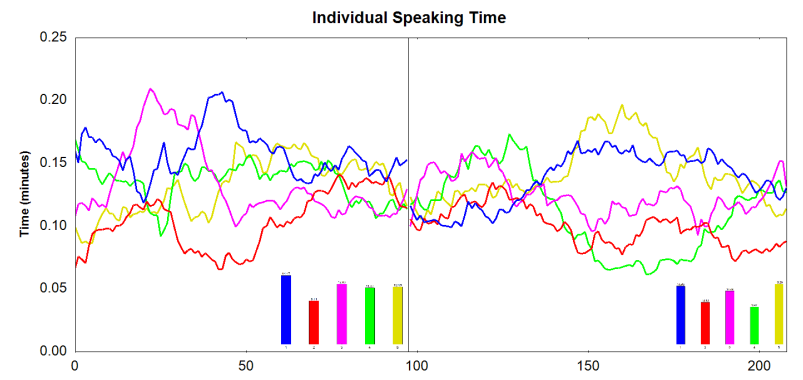

Figure 5: Balanced Participation and No Leadership in Group D

On the contrary, group $\mathrm{C}$ (Fig. 6) is representative for having a single, distinguishable leader (the red participant). The plot clearly shows that the leader was not only speaking the most, but he was also speaking the most at all times during the session. Also, the other participants rarely regulated their contributions, and the count of changes or adjustments in the amount of speech throughout the meeting is the least among all groups.

The behavior of group D persisted throughout all the sessions, be it hidden mode or territory. We take particular interest in this group, for having certain specific characteristics: they were the only group to schedule and attend all the meetings with the table, never losing data and giving more feedback than other groups. We suspect that a high commitment and a very professional organization between

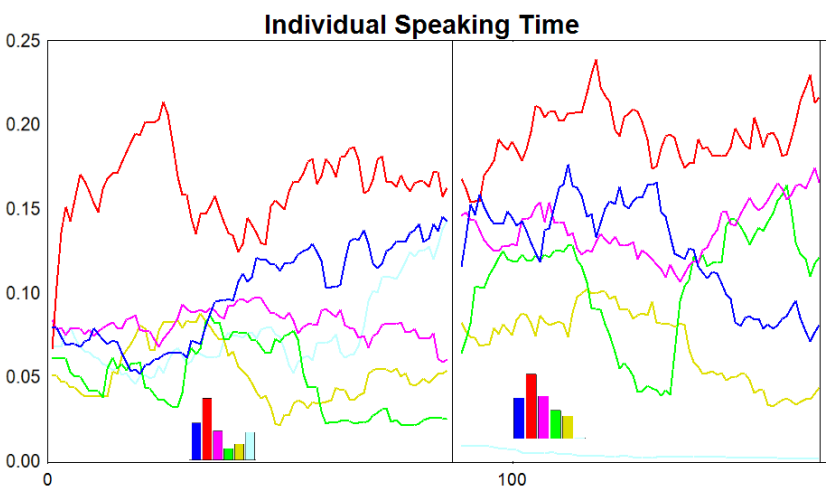

Figure 6: Red participant is the Leader in Group C

the members exists within this group. Also, the behavior of group $\mathrm{C}$ repeated throughout all the sessions, therefore prompting us to assert that the functional aspect of the group relied on that leader (red participant).

\subsubsection{Meeting Phases: a form of regulation}

After analyzing data from meetings in territories mode, we present in Fig. 7 a graph of one meeting with an evident pattern of in-meeting floor turns of four participants, in Group E, occurring during session number 4 (the first one with the territory display). The picture clearly shows a pattern of participants sharing the floor in turns, which could have come as an effect of the territories displayed during the meeting. The longer floor captures leads to assumptions of existence of phases in meetings, which if enforced by the participants, are still a form of participation regulation.

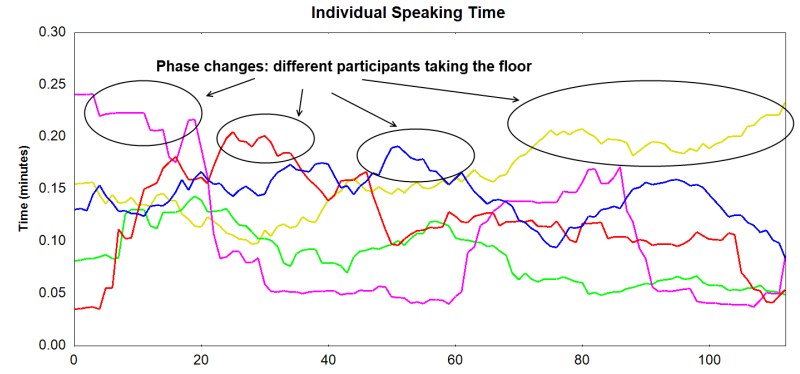

Figure 7: Speech turns within one meetings

This might suggest that since the participants are required to share individual experiences, this group might have defined a strategy to allocate time intervals for each member to share his story and later to discuss about it.

\subsubsection{Turn Takings: more interactivity when territo- ries are visible}

We compared the graphical plots of turn takings in the hidden mode and in the territories mode, observing that typically there is less interactivity in the hidden mode. pairwise lines whose colors point out the number of speech acts transfers from one person to another and where the darker gradient suggests a higher number of transfers.

For group F, This is visible with the larger number of darker lines than in Figure 8. This pattern is less strong for the other groups, but a slight improvement in interactivity 


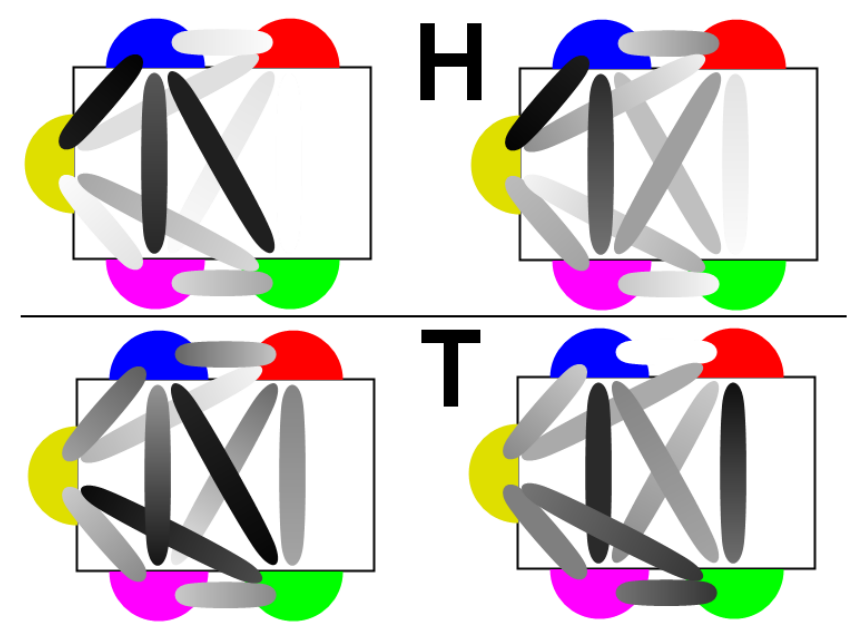

Figure 8: Turn Takings: Hidden vs. Territories

is seen in most of them. An assessment of the effect of interactivity on the quality of interaction would be useful, especially to judge the quantity of speech overlapping (which occurs more in the case of increased interactivity) and its possible detrimental effect. However our technology does not support a reliable enough measurement capacity for such overlappings.

\subsubsection{Effects of the distribution of the Report: there is regulation between meetings as well}

Regarding the availability of the report, by looking at the first sessions, it is our contention that there is an effect from its very first distribution on some people. Data from groups $\mathrm{A}$ and $\mathrm{B}$ shows that the two most and least speaking persons during the first session practically exchanged these roles during the second meeting (Fig. 9). Group A continued this exchange within the 3rd meeting as well, where the red participant came back to speaking more.

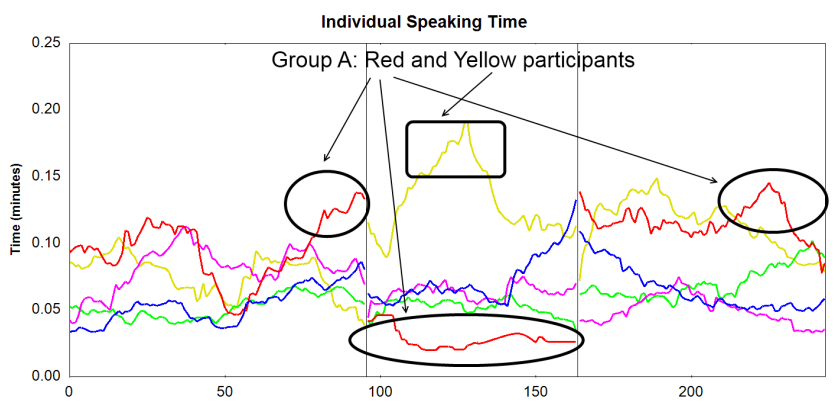

Figure 9: Behavioral Change in Group A

Another type of regulation that we observed and which could come as an effect of distributing the reports, was an inmeeting pair domination in group B throughout all sessions, but with a different pair of participants dominating each meeting (Fig. 10). This result is new and suggests that a distributed pair leadership pattern emerges for this group, probably as a means of structuration of interaction.
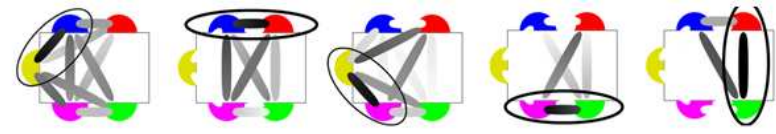

Figure 10: Pair dominance group B

\subsubsection{Group Speaking Time: groups have meetings trademarks and reproducible behavior}

We moved on to analyze the in-meeting group interaction in terms of evolution of total group speaking time (per minute). By analyzing all the available meetings, we concluded that there are 3 patterns of speech in a meeting: a double hill, an increasing interaction, and a decreasing interaction. The patterns observed are completely new and could be used to predict meeting phases, or even meeting signatures for some groups. In the case of groups $\mathrm{D}, \mathrm{E}$, and $\mathrm{F}$ (Fig. 11), the same pattern was present throughout all their encounters therefore suggesting some internal fingerprint of the group.

The other groups did not have such consistency between meetings, but still they had an evolution combining meetings with one of these these three patterns. Group A had a prevalence for the two hills, Group B a majority of sessions in slightly downtrend, and group $\mathrm{C}$ mixed two hills with uptrend.

\subsection{Qualitative Assessment}

Among the questionnaires we sent, some of them were concerned with the use of the table and its perceived impact in the hidden mode (after 3rd sessions), others were about the territories mode (after 5th sessions), and some were concerned with the quality and utility of the report (around 4th sessions). Open feedback was gathered by the coordinator in a general debriefing reunion around the time of the 7 th sessions. A first observation about the questionnaires is that we received a rather low overall response rate (between $32 \%$ and $58 \%$ ), probably due to the participants not taking the time to fill them in.

\subsubsection{Individual sessions Impressions}

The questionnaire for the hidden mode evaluated the reaction at the end of the meeting, when switching to the territories. We received a low number of replies. In all cases there was a debriefing discussion of at least one minute about the participation, interaction, and the table display. Half the respondents were surprised by the result, the other half foresaw the territories to look like they did.

In the territories questionnaire, we asked how often they looked at the table during the session, what they saw, and if that prompted them change their behavior. We received a much higher number of replies for this questionnaire, $77 \%$ of the respondents reporting looking at the table display during the session, and of these, $40 \%$ identified themselves as speaking more than others, all of them claiming to give the floor away from that moment on. When asked to evaluate if the changes in the behavior of the others were perceivable (adjusting to speak more or speak less), half of the replies were positive. However, among those who identified themselves as speaking less, almost no one reported taking any immediate initiative of balancing by speaking more.

The reports questionnaire was distributed online, decoupled from the meetings dates, with a rather medium re- 


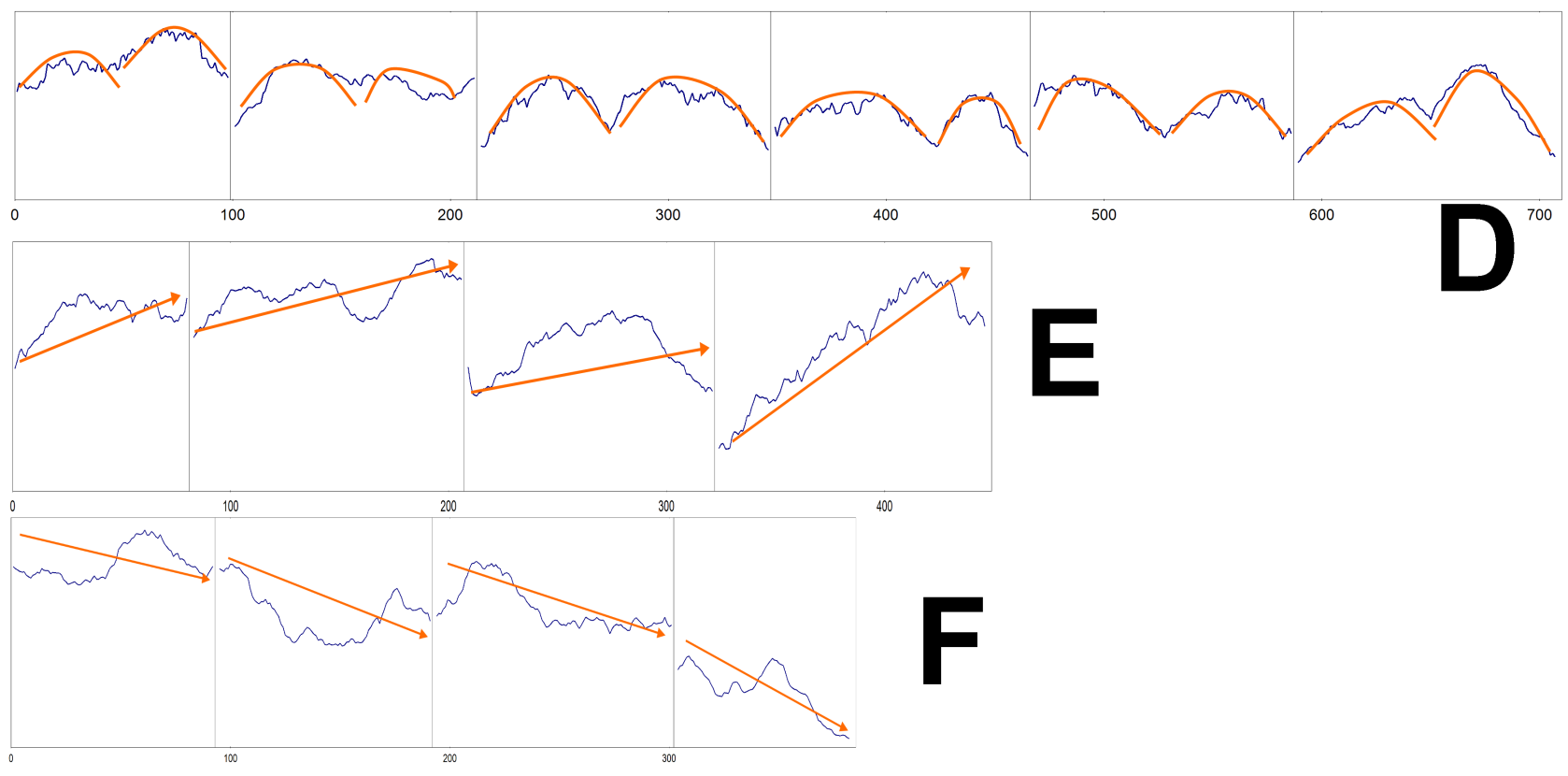

Figure 11: Three Group Speech Patterns emerge across sessions: Double Hill, Increasing, Decreasing

sponse rate compared to the other questionnaires. Many participants were interested to get more in-depth on the analysis and explanations of graphics, which suggests that the idea of distributing a report was well received. $83 \%$ of the replies qualified the report as useful or extremely useful, and they stated they do use it to reflect on their behavior as a guide for the next meetings. There were demands for group evolution measures as well. We interpret that continuing to offer reports and expanding or improving the information in them can have a positive effect on individual adaptation towards group cohesion and performance.

\subsubsection{System Feedback from Users: new findings}

We received the highest degree of responses in the open feedback, and we classified the remarks into 3 categories: positive $(39 \%)$, neutral or observing no effects $(44 \%)$ and negative $(16 \%)$, with respect to the perceived value of the Reflect Table and meeting analysis.

Among the positive feedback, we read statements commending the utility of the system for its designed purpose, users reporting adjusting their behaviors when their territories were too large, as well as the system itself being labeled as a reference for scheduling meetings by one of the teams (the constraint of a specific room made it easier for them to schedule the meetings), while others viewed it as an objective technology promoting participation from all sides.

The sum of the neutral feedback was filled by reports that attendants did not perceive the table to be necessarily the cause of in-meeting regulation, but rather relied on self-designed group norms for that. We can think that most of these replies were from people who did not find themselves speaking a lot more than others. Participants did mention however that they took note of their evolution of behavior throughout the meetings by looking at the reports.

We classified the negative feedback as the comments that were against having the technology or using it. We collected remarks of people who were not convinced that the tech- nology works, especially because of the existing wider side in the table, and considered that showing this data (which they perceived as inaccurate) was counterproductive for the group. One person complained that we don't count active listening as participation, another one expressed concern that the territories sizes are never surprising and thus the value of having them is discounted by the group. Another one complained that the link between the color and his identity (due to the construction of the territories, which originate in front of each user) affected his reputation when he intentionally did not want to contribute to a part of the discussion (he perceived that his intention of non-participating was actually revealed by the table to the others, whereas he would have liked to conceal it). The aspects revealed will be deeply considered in our future work.

By corroborating these findings with the other feedback and the quantitative analysis, we can well believe that even if there was average enthusiasm expressed by the participants in the questionnaires, the system did well in fulfilling the goal of regulating participation over time and helping the teams become cohesive and productive.

\subsection{Limitations and Discussion}

The uncontrolled nature of the study brings about limitations and discussions regarding the results obtained. Since we had no access to content and measurements of process variables, or clues about the unfolding of social rapports between individuals within groups, and also there is only scarce assessment of sociocognitive aspects of interactions (that might have an impact on the speech time behavior), it is difficult to strongly claim that our system and technology was all that it took to balance participation.

We are convinced that the principles used for the design of such support systems are adequate and the support the tools bring to the social cognition and interactions of groups in meetings are helpful, however we cannot rule out that 
certain characteristics of the context of the study did not carry effects into the results. For example:

- Specific characteristics of the groups composition: not knowing each other prior to the creation of the groups can be a factor of increased mutual respect and predisposition for speech time sharing;

- Type of meetings: trainings based on sharing experiences may be more prone to balanced participation than other types of meetings, such as information or decision making where imbalanced participation may be natural. We restate that our technology is not normative, hence it only provides knowledge that the participants are free to use the way they feel appropriate;

- Type of attendance: having peers in an organizational context is certainly a distinct situation with respect to the outcome, when compared to groups that are composed of people with different hierarchical authorities.

We would therefore limit our claims to the context of these particular aspects of the study. However, within this framework, we do believe that the cognizance provided by our system has a positive effect even for top level managers.

\section{FUTURE}

The added value brought by the Reflect Table and ReflectVisualizations system is encouraging us to continue to pursue research in the field of our interest, using these tools or similar ones, in several directions.

One of them is to upgrade the technology above the Reflect Table's principles by optimizing the parameters measured and creating other tools that mitigate some of the existing limitations. We are creating a dematerialized version of the Reflect Table, in the form of software running on mobile devices aiming to retain similar features. With the emergence and omniscience of the iPhones, iPads, Android and similar technologies, we can leverage their adoption to port our work on them, therefore eliminating the drawback of location-dependency on a heavy table, enabling parallel meetings support, centralized data processing, and better analysis and reporting. We can change Reflect Visualizations to be an even more integrated module. The pursuit can follow either of two directions: using a single iPad and detecting who is speaking (based on a calibration and fingerprinting of the voices of the participants), or using one iPad per participant, and synchronizing the data generated. The main challenges are, in both cases, the correct detection of the speaker, with an additional requirement for consistency of generated data timestamps in the second scenario.

The added support for in-depth post-meeting analysis of the encounter provides insight on group evolution and evolution of individual behavior throughout multiple meetings in the same group. We expect more group evolution to unfold in time, and further research on future sessions will help us better see the effects of technologies in these meetings. The next step is to perform more thorough analysis of group evolution, by evaluating more precise leadership traits, and also emerging norms and types of self-organization within these groups.

\section{CONCLUSIONS}

We presented a meeting toolkit rooted in the Reflect Table and extended with new functionalities of the ReflectVisualizations software for meetings analysis. We devised a study in an uncontrolled environment of top level managers, in the context of training meetings that were designed to achieve a good level of mutual knowledge between participants by exchanging individual past experiences, where they used the Reflect Table and the reports generated by the ReflectVisualizations software. Previous studies of the Reflect Table done with students showed that awareness about speech time has an effect of equilibrating participation within a meeting, and we wanted to evaluate whether the same effect would be obtained in a real life setting, rather than a laboratory experiment, either as in-meeting or over multiple meetings. The results of the current study are light for in-meeting balancing effect, but are very positive in terms of balancing over time after more meetings, showing that the top level managers do use self-regulation when becoming aware of their speech, with a more pronounced effect observed after several meetings.

The main contribution of this work is that it extends the study of awareness tools in meetings, to show their positive results in real world as well. With the creation of the new tools that expand the existing Reflect Table for meetings analysis, we make available a complete system that is robust enough to be used in measuring and assessing long term groups evolution, in any given organizational habitat where a team, task force or contingent performs a collaborative face to face activity, and where contribution from individual members is valued.

\section{ACKNOWLEDGMENT}

We are strongly indebted to Banque Privée Edmond de Rothschild, Geneva, Switzerland, member of the Edmond de Rothschild Group, for the sponsorship and support of our research.

\section{REFERENCES}

[1] K. Bachour. Augmenting Face-to-Face Collaboration with Low-Resolution Semi-Ambient Feedback. PhD thesis, EPFL, Lausanne, 2010.

[2] K. Bachour, F. Kaplan, and P. Dillenbourg. Reflect : An interactive table for regulating face-to-face collaborative learning. In Lecture Notes in Computer Science. Springer, Berlin / Heidelberg, 2008.

[3] K. Bachour, F. Kaplan, and P. Dillenbourg. An interactive table for supporting participation balance in face-to-face collaborative learning. IEEE Transactions on Learning Technologies, 3:203-213, 2010.

[4] K. Bachour, H. Seiied Alavi, F. Kaplan, and P. Dillenbourg. Low-resolution ambient awareness tools for educational support. In The Future of HCI And Education, 2010. CHI 2010 Workshop: The Future of HCI And Education, Atlanta, Georgia, USA, April 11, 2010.

[5] T. Bergstrom and K. Karahalios. Conversation clock: Visualizing audio patterns in co-located groups. In Proceedings of the 40th Annual Hawaii International Conference on System Sciences, HICSS '07, pages 78-, Washington, DC, USA, 2007. IEEE Computer Society. 
[6] T. Bergstrom and K. Karahalios. Visualizing colocated conversation feedback. IEEE Tabletop, $1: 165-178,2007$.

[7] K. Bettenhausen and J. K. Murnighan. The emergence of norms in competitive decision-making groups. Administrative Science Quarterly, 30(3):pp. 350-372, 1985.

[8] P. Boersma and D. Weenink. Praat: doing phonetics by computer (version 5). Glot International, 5:341-345, 2001.

[9] E. G. Cohen. Restructuring the classroom: Conditions for productive small groups. Review of Educational Research, 64(1):1-35, 1994.

[10] J. DiMicco and W. Bender. Group reactions to visual feedback tools. In Y. de Kort, W. IJsselsteijn, C. Midden, B. Eggen, and B. Fogg, editors, Persuasive Technology, volume 4744 of Lecture Notes in Computer Science, pages 132-143. Springer Berlin / Heidelberg, 2007.

[11] C. Ellis, P. Barthelmess, B. Quan, and J. Wainer. Neem: An agent based meeting augmentation system, 2001.

[12] J. R. Hackman. Group influences on individuals in organizations. Handbook of industrial and organizational psychology, 3:199-267, 1992.

[13] C. Hoyles. What is the point of group discussion in mathematics? Educational Studies in Mathematics, 16:205-214, 1985. 10.1007/BF02400938.

[14] G. P. Huber. A theory of the effects of advanced information technologies on organizational design, intelligence, and decision making. The Academy of Management Review, 15(1):pp. 47-71, 1990.

[15] S. Hunter, P. Maes, S. Scott, and H. Kaufman. Memtable: an integrated system for capture and recall of shared histories in group workspaces. In Proceedings of the 2011 annual conference on Human factors in computing systems, CHI '11, pages 3305-3314, New York, NY, USA, 2011. ACM.

[16] T. Kim, A. Chang, L. Holl, and A. Pentland. Meeting mediator: Enhancing group collaboration using sociometric feedback. In Proceedings of the 2008 ACM conference on Computer supported cooperative work, 2008.

[17] A. Nijholt, R. Rienks, and D. Reidsma. Online and off-line visualization of meeting information and meeting support. The Visual Computer, 1:965-976, 2006.

[18] N. Romano Jr and J. Nunamaker Jr. Meeting analysis: Findings from research and practice. In Proceedings of the 34th Annual Hawaii International Conference on System Sciences ( HICSS-34)-Volume 1 - Volume 1, HICSS '01, pages 1072-, Washington, DC, USA, 2001. IEEE Computer Society.

[19] G. Salomon and T. Globerson. When teams do not function the way they ought to. International Journal of Educational Research, 13:89-99, 1989.

[20] T. Skog, S. Ljungblad, and L. E. Holmquist. Between aesthetics and utility: designing ambient information visualizations. In Proceedings of the Ninth annual IEEE conference on Information visualization, INFOVIS'03, pages 233-240, Washington, DC, USA, 2003. IEEE Computer Society.

[21] S. Streng, K. Stegmann, H. Hussmann, and F. Fischer. Metaphor or diagram?: comparing different representations for group mirrors. In Proceedings of the 21st Annual Conference of the Australian Computer-Human Interaction Special Interest Group: Design: Open 24/7, OZCHI '09, pages 249-256, New York, NY, USA, 2009. ACM.

[22] J. Webster, Murray and J. Driskell, James E. Beauty as status. American Journal of Sociology, 89(1):pp. 140-165, 1983. 